\title{
Aerodynamic response of an airfoil section undergoing pitch motion and trailing edge flap deflection: a comparison of simulation methods
}

Bergami, Leonardo; Riziotis, Vasilis A. ; Gaunaa, Mac

Published in:
Wind Energy

Link to article, DOI:

10.1002/we.1759

Publication date:

2015

Document Version

Early version, also known as pre-print

Link back to DTU Orbit

Citation (APA):

Bergami, L., Riziotis, V. A., \& Gaunaa, M. (2015). Aerodynamic response of an airfoil section undergoing pitch motion and trailing edge flap deflection: a comparison of simulation methods. Wind Energy, 18(7), 1273-1290. https://doi.org/10.1002/we.1759

\section{General rights}

Copyright and moral rights for the publications made accessible in the public portal are retained by the authors and/or other copyright owners and it is a condition of accessing publications that users recognise and abide by the legal requirements associated with these rights.

- Users may download and print one copy of any publication from the public portal for the purpose of private study or research.

- You may not further distribute the material or use it for any profit-making activity or commercial gain

- You may freely distribute the URL identifying the publication in the public portal 


\title{
Aerodynamic response of an airfoil section undergoing pitch motion and trailing edge flap deflection: a comparison of simulation methods
}

\author{
Leonardo Bergami*, Vasilis A. Riziotis ${ }^{\ddagger}$, Mac Gaunaa* \\ * DTU Wind, Technical University of Denmark, Denmark. \\ $\ddagger$ Fluids Department, National Technical University of Athens, Greece.
}

leob@dtu.dk

\begin{abstract}
This is the pre-peer reviewed version of the following article: Bergami, Leonardo, Vasilis A. Riziotis, and Mac Gaunaa. Aerodynamic Response of an Airfoil Section Undergoing Pitch Motion and Trailing Edge Flap Deflection: A Comparison of Simulation Methods. Wind Energy, May 2014, Early View. doi:10.1002/we.1759, which has been published in final form at Wiley Online Library
\end{abstract}

\begin{abstract}
The study presents and compares aerodynamic simulations for an airfoil section with an adaptive trailing edge flap, which deflects following a smooth deformation shape. The simulations are carried out with three substantially different methods: a Reynolds Averaged Navier-Stokes solver, a viscous-inviscid interaction method with single and double wake implementations, and an engineering dynamic-stall model suitable for implementation in aeroelastic codes based on Blade Element Momentum theory. The aerodynamic integral forces and pitching moment coefficients are first determined in steady conditions, at angles of attack spanning from attached flow to separated conditions, and accounting for the effects of flap deflection. The paper characterizes then the dynamics of the unsteady forces and moments generated by the airfoil undergoing harmonic pitching motions, and harmonic flap deflections. The dynamic responses produce important variations of the aerodynamic coefficients over their corresponding steady values. The dynamics characteristics of the unsteady response are predicted with an excellent agreement among the investigated methods in attached flow conditions, both for airfoil pitching and flap deflection. For higher degrees of flow separation, the methods still depict similar overall dynamics, but larger discrepancies are reported, especially for the simpler engineering method.
\end{abstract}

\section{Introduction}

Several research projects have highlighted the potential benefits of a new generation of wind turbine rotors, which would allow for enhanced active load alleviation by including active aerodynamic devices distributed along the blades $[1,2]$. Active aerodynamic devices are able to alter the aerodynamic forces locally along the blade span by modifying the geometry of the airfoil section; 
they thus allow to vary the aerodynamic loading on the whole blade, without acting on its pitch angle. Particularly promising results in terms of fatigue load alleviation have been reported by simulations considering adaptive trailing edge flap (ATEF) devices $[3,4,5,6]$. The adaptive trailing edge flap modifies the geometry of the airfoil by deflecting the aft portion of its camber-line; Troldborg et al. [7] have shown that deflections following a smooth continuous deformation shape return better aerodynamic performances compared to a classic rigid flap rotating around its hinge point.

The presence of the active flaps poses new challenges to the aerodynamic models used in the design and aeroelastic simulation of the turbine response; the steady aerodynamic forces and moment on the blade sections as well as their unsteady dynamics will in fact depend not only on the section angle of attack, but also on the flap deflection. Previous studies have addressed the problem of simulating unsteady aerodynamic forces on an airfoil section with flaps by using, among others, Navier-Stokes solvers [8, 9], panel code methods [10], viscousinviscid interaction models $[11,12]$, and simpler engineering methods, which considered either quasi-steady approximations [13], or attached flow models $[3,14]$, or dynamic-stall type of models $[15,5]$. On account of their lower computational requirements, engineering methods have often been integrated in Blade Element Momentum (BEM) based aeroelastic codes, allowing thus to simulate the full response of a turbine with active flaps.

The paper considers three state-of-the-art methods to simulate the integral aerodynamic forces and moment coefficients of a $2 \mathrm{D}$ airfoil section undergoing pitching motion, and trailing edge flap deflections. The methods are, in decreasing order of computational requirements: EllipSys 2D, a Reynolds Averaged Navier-Stokes (RANS) solver [9]; NTUA viscous-inviscid interaction method [16] with a double wake, and a single wake implementation; ATEFlap, a dynamic-stall engineering model [17].

The aim is to characterize the unsteady aerodynamic response of the airfoil to changes in the angle of attack or the flap deflection, and to compare the responses simulated by the three methods. Similarities and differences among the simulated responses provide an indication of the codes modeling performances; in particular, the comparison of the simpler ATEFlap dynamic-stall model against more complex methods will serve to validate the model capabilities, and to highlight its limitations. The ATEFlap model, thanks to its low computational requirements, can be conveniently integrated in a BEM-based aeroelastic simulation tool, and thus employed to design and assess the response of a wind turbine with active flaps. The paper further develops the code comparison task carried out initiated within the frame of the UpWind European project, work package 2 "Aerodynamics and aero elastics" [18].

The following section briefly describes the investigated methods, and their prominent characteristics, thorough descriptions are provided in the bibliographic references. Section 3 lists the specification of the test case considered in the simulations, and describes the airfoil and trailing edge flap set-up. Aerodynamic forces for different angles of attack and flap deflections are first computed with EllipSys and the NTUA codes in steady conditions; the results, presented in section 4, serve as input to the ATEFlap model. Simulations are then performed for the airfoil undergoing prescribed harmonic pitching motion, and harmonic flap deflection; the aerodynamic response is simulated at different mean angles of attack, covering attached flow conditions, separation onset, and stalled flow, 
section 5 .

\section{Computational methods}

\section{$2.1 \quad$ EllipSys}

EllipSys 2D is a CFD (Computational Fluid Dynamic) code originally developed by Michelsen [19, 20], and Sørensen [21]. The code solves the incompressible Reynolds Averaged Navier-Stokes (RANS) equations in general curvilinear coordinates, using a multiblock finite volume discretization. The computations shown in this work are all carried out under the assumption of fully turbulent flow, and applying the k- $\omega$ Shear Stress Transport (SST) eddy-viscosity turbulence model from Menter [22].

The motion of the airfoil is simulated by moving the computational grid, and accounting for the additional fluxes that are generated as the grid cells vertices are displaced. The flap deflection is modeled through a grid morphing routine [9], where the position of the grid points for an arbitrary flap deflection are determined by linear interpolation of the two meshes generated with the flap at maximum upwards and downwards deflection $\left( \pm 5^{\circ}\right.$ in this study); the additional fluxes caused by the displacement of the cell vertices are also accounted for.

Among the investigated methods, the EllipSys RANS solver is the one with the highest computational requirements: simulations of a 10 seconds time series of the aerodynamic forces on a typical 2D airfoil section, as the one presented here, require approximately 3 minutes when parallelized on 4 CPUs.

\subsection{NTUA $1 \mathrm{~W}$ and NTUA $2 \mathrm{~W}$}

NTUA $1 \mathrm{~W}$ and NTUA 2W are viscous-inviscid interaction codes that have been developed at NTUA $[23,12,16]$. In both codes the potential flow part is simulated by singularity distributions along the airfoil geometry and along the wake (sources and vortices). The wake is represented by vortex particles (point vortices), which are allowed to freely move with the local flow velocity (free wake approach). The viscous flow solution is obtained by solving the unsteady integral boundary layer equations. The coupling of the two sets of equations is achieved through a transpiration velocity distribution along the airfoil surface that represents the mass flow difference over the boundary layer height between the real viscous flow and the equivalent inviscid flow. The boundary layer equations are discretized using finite differences, and the final set of nonlinear equations (potential and boundary layer) is solved simultaneously using Newton-Rapshon algorithm. The boundary layer solution is supplemented by a transition prediction model based on the $e^{N}$ spatial amplification theory, and by a dissipation closure equation for the maximum shear stress coefficient $\mathrm{Ct}$ over the turbulent part.

The main difference between the NTUA $1 \mathrm{~W}$ and NTUA $2 \mathrm{~W}$ codes is that in NTUA 1W the boundary layer equations are integrated over the whole airfoil surface and the trailing edge wake, while in NTUA 2W they are only solved up to the position where flow separation takes place. Thereafter, a second vortex sheet is shed from the separation location and interacts with the trailing edge vortex sheet; together they form the separation bubble. By introducing this 
second vortex sheet, convergence of the solution can be achieved even in deep stall conditions. This is the main advantage of the double wake approach against conventional boundary layer methodologies.

Among the different methods tested in the paper, NTUA 1W and NTUA $2 \mathrm{~W}$ models are intermediate in terms of computational cost. A 10 seconds simulation of a 2D airfoil is resolved in about two minutes on a single CPU. Although the simulation gets slower, as the number of particles in the wake increases, hybrid wake acceleration techniques have been developed with the aim to keep computational effort constant [24].

\subsection{ATEFlap}

The ATEFlap is an engineering model, which couples a potential flow solution with a Beddoes-Leishmann-type dynamic stall model. The model develops, and partially amends the shortcomings of the dynamic-stall model presented in Andersen et al. [15]; a more detailed description is reported in Bergami and Gaunaa [17].

The potential flow part of the model is based on Gaunaa's [25] model for a thin airfoil undergoing arbitrary motion and camber-line deformation; the dynamics effects on the circulatory forces are described through a superposition of indicial response functions of the Wagner type. The indicial response function is formulated in exponential terms to allow for an efficient time integration scheme, and the function coefficients are tuned to fit the indicial response of the investigated airfoil, table 1, as it differs from Jones's standard flat-plate response [26].

Flow separation dynamics are represented by a Beddoes-Leishmann type dynamic stall model, as described in Hansen et al. [27], where the total circulatory lift force is computed as a weighted sum of a fully attached contribution, and a fully separated one. The separation dynamics are enclosed in the weight factor of the sum, which result from a sequence of two first-order low-pass filters, whose time constants depend on two non-dimensional parameters $\tau_{P}$ and $\tau_{B}$ $[27,17]$. The values used in the computations are reported in table 1.

\begin{tabular}{|cccc||cc|}
\hline$A_{i}$ & 0.1784 & 0.07549 & 0.3933 & $\tau_{P}$ & 1.5 \\
\hline$b_{i}$ & 0.8000 & 0.01815 & 0.1390 & $\tau_{B}$ & 6.0 \\
\hline
\end{tabular}

Table 1: Computation set-up for the ATEFlap dynamic-stall model. Indicial lift response function coefficients for NACA 64-418 airfoil, and dynamic stall non-dimensional time parameters.

The model requires as input the airfoil steady integral forces and moment coefficients at various angles of attack, as well as the coefficient variations caused by steady flap deflections. In the study, three set of steady input referring to the same airfoil and flap configuration are considered: one generated from steady computations with EllipSys 2D, and two sets retrieved from steady simulations with the NTUA code, using either the single or the double wake configuration.

Simulations with the ATEFlap model have very low computational requirements, rendering the model particularly attractive for implementation in time marching aeroelastic simulation codes [17]; as an indication, a 10 seconds simulation of unsteady forces on a $2 \mathrm{D}$ airfoil section is resolved in less than one second on a single CPU. 


\section{Test case}

The airfoil considered in the study has a NACA $64-418$ profile, and is fitted with an adaptive trailing edge flap, which extends for the last $10 \%$ of the chord. At null flap deflections, the airfoil has the standard NACA 64-418 profile, while positive flap angles corresponds to a downwards deflection of the trailing edge (increased lift), and vice-versa for negative flap angles, figure 1.

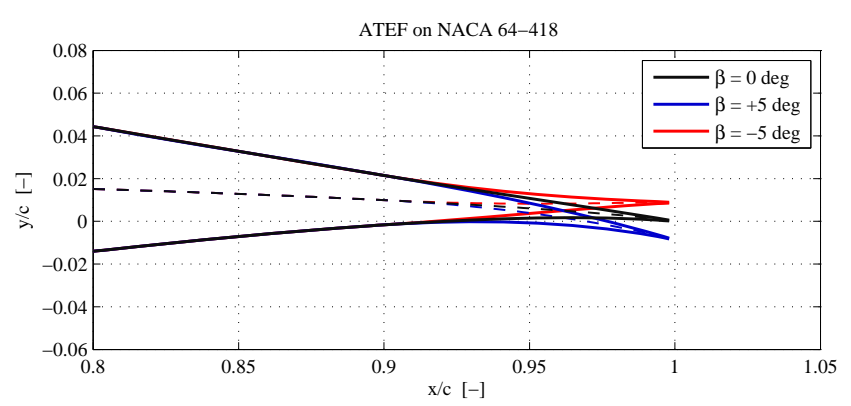

Figure 1: Camber-line deformation corresponding to flap deflections of $\pm 5^{\circ}$ on the investigated NACA 64-418 airfoil profile.

The deflection of the flap is modeled as a deformation of the camber-line, leaving the airfoil thickness unchanged. The camber-line points are displaced normally to the chord-line by a distance $\Delta y_{\text {camb }}=\beta \cdot y_{f l}$, where $\beta$ is the flap deflection (in degrees), and the function $y_{f l}$ describes the flap deflection shape, i.e. the camber-line variation for a unitary flap deflection. The deflection shape for a unit chord length airfoil is defined as a circular arc starting at $90 \%$ of the chord length; the radius of the circle is set so that the line connecting the point on the arc at the trailing edge with the flap starting point forms an angle of 1 degree with the undeformed chord-line, figure 1:

$$
y_{f l} / c \quad \begin{cases}y_{f l} / c=0.0 & \text { for } x / c<0.9 \\ y_{f l} / c=\sqrt{R_{c}^{2}-(x / c-0.9)^{2}}-R_{c}^{2} & \text { for } x / c \geq 0.9\end{cases}
$$

where the radius of the circular arc is given by

$$
\begin{aligned}
R_{c} & =\frac{0.1^{2}+\delta_{y}^{2}}{2 \delta_{y}}, \\
\delta_{y} & =0.1 \tan (1 \cdot \pi / 180) .
\end{aligned}
$$

Simulations are carried out for the airfoil undergoing harmonic variations of the angle of attack (aoa), obtained by prescribed pitch motion with respect to a rotation axis located at the quarter-chord of the airfoil. A combination of different amplitudes and mean angles of attack is considered, table 2, spacing from fully attached to stalled flow conditions. Additional simulations evaluate the response to prescribed harmonic flap deflections of various amplitudes, table 2 , and with the airfoil fixed at the same mean angles of attack as specified before; note that, in this study, the airfoil angle of attack is always defined with respect to the chord-line corresponding to the undeflected flap.

The simulations are replicated for harmonic variations with three reducedfrequencies $k$, also listed in table 2 . The reduced-frequency is a dimensionless 
quantity, which gives an indication of the degree of 'unsteadiness' of the aerodynamic problem; it is defined as

$$
k=\frac{\omega b_{h c}}{U_{\infty}}
$$

where $\omega$ is the frequency of the harmonic variations, $b_{h c}$ is the half-chord length, and $U_{\infty}$ the free-stream flow speed. The investigation comprises reduced frequencies that fall within the intervals of what are typically defined as quasisteady $(k=0.02)$, unsteady $(k=0.1)$, and highly-unsteady $(k=0.5)$ conditions; the first two values corresponds to the reduced frequencies typically encountered on wind turbine blades in relation to the rotational frequency. All the simulations are carried out with a Reynolds number of six millions based on the airfoil chord.

\begin{tabular}{|cc|l|}
\hline Mean Aoa & {$[\mathrm{deg}]$} & $0,8,12,16,18$ \\
Aoa ampl. & {$[\mathrm{deg}]$} & $0.5,1.0,2.5$ \\
Flap ampl. & {$[\mathrm{deg}]$} & $1.0,2.0,5.0$ \\
Red.frq. $k$ & {$[-]$} & $0.02,0.1,0.5$ \\
\hline
\end{tabular}

Table 2: Test matrix for the code comparison unsteady simulations. Each combination of mean angle of attack and oscillation amplitude (for either the angle of attack or the flap) is replicated for each of the three reduced frequencies.

\section{Steady aerodynamic response comparison}

The steady aerodynamic responses of the airfoil and flap are simulated with EllipSys, and the NTUA codes, with single $(1 \mathrm{~W})$, and double wake $(2 \mathrm{~W})$ configurations; the double wake implementation is considered exclusively in separated flow conditions, for angles of attack from 11 to 22 degrees. The steady data provide a baseline indication of the unsteady loops mean values; as the three methods return different steady responses, each method will provide a separate set of steady input data for the ATEFlap model.

The steady responses are presented first for the airfoil with the flap fixed in neutral position, thus corresponding to a standard NACA 64-418 profile; the steady effects of flap deflection are then reported as variations around the baseline values for the integral aerodynamic forces and moment at different angles of attack.

\subsection{Baseline airfoil}

The steady lift coefficients returned by EllipSys and NTUA $1 \mathrm{~W}$ are in very good agreement in the attached flow linear region, figure 2(a); the curves have nearly the same slope, and only a small offset on the angle of attack corresponding to zero lift. The agreement deteriorates at higher angles of attack, where, due to more pronounced flow separation, the thin wake assumption made in the single wake method is no longer valid, and the NTUA $1 \mathrm{~W}$ code returns higher lift values. The double wake method returns slightly lower lift coefficients, with a marked dip around $18^{\circ}$ not seen in the EllipSys results. 


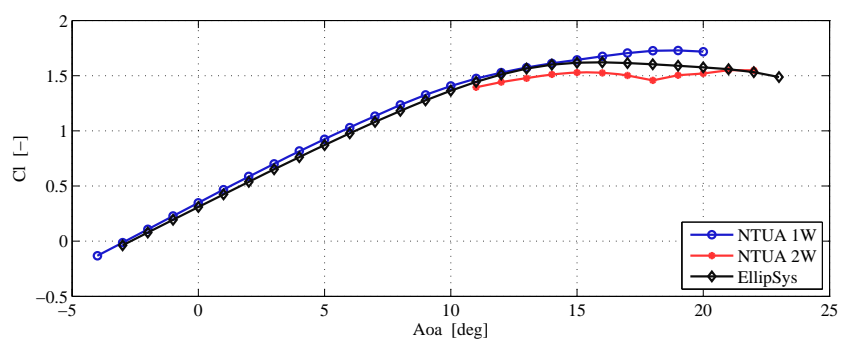

(a) Lift Coefficient

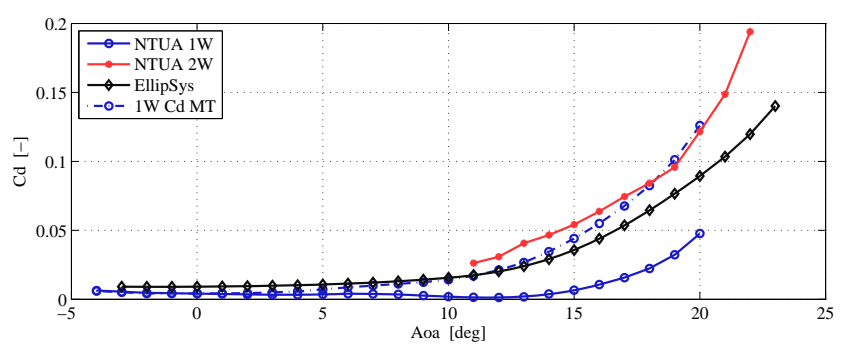

(b) Drag Coefficient. The steady drag computations based on momentum theory for the NTUA 1W result are included for comparison $(1 \mathrm{~W}$ Cd MT).

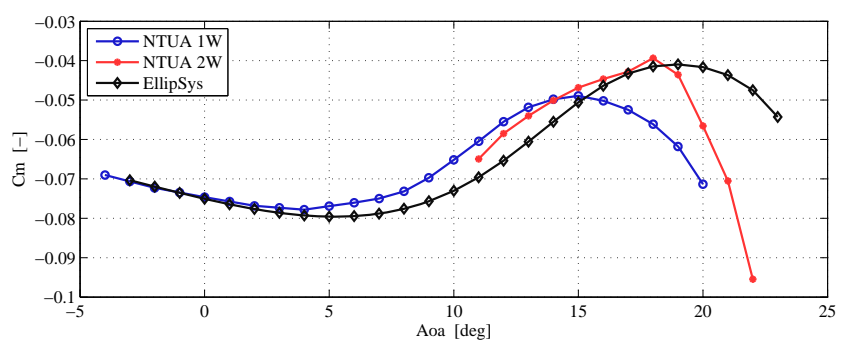

(c) Moment Coefficient

Figure 2: Steady aerodynamic response for the NACA 64-418 and flap in neutral position.

The prediction of drag forces, figure $2(\mathrm{~b})$, is more problematic for the boundary layer methods, as the drag force returned by direct pressure integration is biased by the suppression of the streamline curvature effects [12]. The bias is particularly marked in the NTUA 1W response, which predicts very low drag coefficients, initially decreasing with the angle of attack. The drag coefficients returned by the momentum equation applied to a control volume around the airfoil are in better agreement with the CFD results, although the drag increase at higher angles of attack is more marked, as shown by the dashed blue line in figure 2(b); a steep drag increase at high angles of attack is also reported in the NTUA 2W results, red line in figure 2(b).

The three codes agree in predicting negative ('nose-down') aerodynamic moments, figure 2(c) and display similar trends of the moment coefficient versus angle of attack, although, especially at higher angles, the coefficient values predicted by the codes are rather different. 


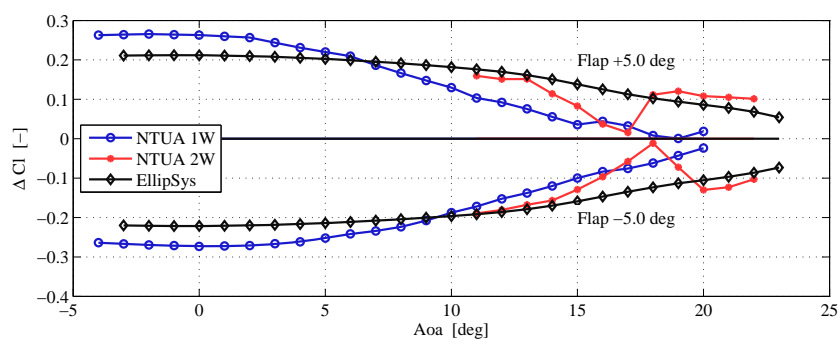

(a) Lift Coefficient

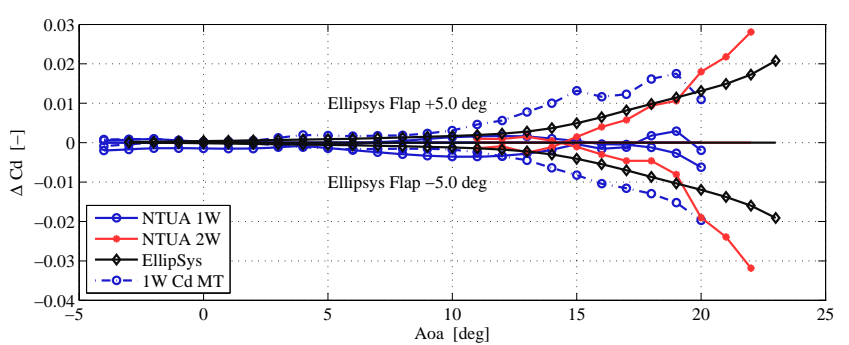

(b) Drag Coefficient. The steady drag computations based on momentum theory for the NTUA $1 \mathrm{~W}$ result are included for comparison (1W Cd MT).

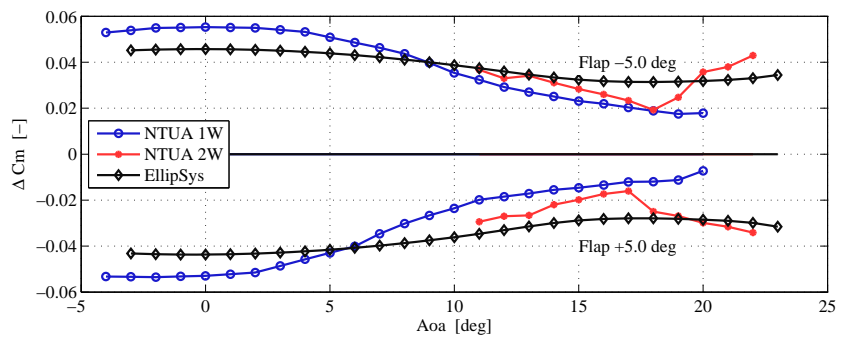

(c) Moment Coefficient

Figure 3: Steady aerodynamic response for flap deflection. The steady effects are expressed in terms of variations from the aerodynamic coefficients of the airfoil with undeflected flap.

\subsection{Trailing edge flap variations}

The steady aerodynamic responses to trailing edge flap deflection are presented as variations of the steady coefficients from the baseline airfoil with undeflected flap.

The maximum lift increase predicted by EllipSys for $+5^{\circ}$ flap deflection is approximately equivalent to an increase of angle of attack of $1.8^{\circ}$, figure $3(\mathrm{a})$. NTUA $1 \mathrm{~W}$ predicts higher maximum lift variations (equivalent to a $2.3^{\circ}$ increase in angle of attack), and a marked decline of the flap effects with increasing angle of attack, especially for positive flap deflections: at $10^{\circ}$ angle of attack the lift variation is already half the maximum one. The decline predicted by EllipSys is smoother, the flap effects are halved above $17^{\circ}$ aoa, and nearly symmetric for positive and negative flap deflections. For higher angles of attack, NTUA 2W returns steady lift variations that are on average closer to EllipSys results, but 
displays a marked dip around $18^{\circ}$ aoa, which has no correspondence in EllipSys, figure $3(\mathrm{a})$.

The drag predictions from direct pressure integrations of the boundary layer methods are affected by the same bias discussed in the previous section, and the single wake code returns a decrease in drag for positive flap deflections, figure 3(b). The results based on the momentum theory are closer to EllipSys, and both methods indicate that for lower angles of attack the flap deflection does not involve significant drag variations. As the angle of attack is increased and the flow starts to separate, the steady drag variation produced by the flap is increased, rather mildly according to EllipSys, whereas a steeper increase of the drag penalty is predicted by the NTUA $1 \mathrm{~W}$ momentum theory and NTUA $2 \mathrm{~W}$ results, dashed blue and red lines in figure 3(b).

The variation in the aerodynamic moment, figure $3(\mathrm{c})$, resemble the variations in the lift coefficient, with NTUA $1 \mathrm{~W}$ predicting larger variations at low angles of attack, but also a steeper decrease. Note that a positive flap deflection, which would displace the trailing edge downwards, results in an increase in lift and drag, and in a negative (i.e. 'nose-down') aerodynamic moment.

\section{Unsteady response comparison}

\subsection{Harmonic pitching motions}

The simulation capabilities of the codes are evaluated in dynamic conditions by first comparing the unsteady aerodynamic responses in the ordinary case of an airfoil undergoing harmonic pitch variations, while the flap is fixed to its neutral position. The responses from the ATEFlap model consider three sets of input data, which are generated from the steady responses computed by, respectively, EllipSys, NTUA 1W, and NTUA 2W. As the drag based on momentum theory is not available in dynamic conditions, the NTUA $1 \mathrm{~W}$ drag data refer to the pressure integration results, and will reflect the biases already observed in the steady responses.

The codes show an excellent agreement when simulating the dynamics of the unsteady lift force in attached flow conditions, figure 4. All the methods characterize the unsteady lift with counter-clockwise loops (the loop direction is marked in the plots by the sequence of a larger and a smaller mark), with an effective slope slightly below the steady curve one; the differences in lift coefficients observed in the steady simulations, fig. 2(a), is reflected in the offset between the loops. The ATEFlap engineering model performs very well, and returns unsteady lift responses that overlie the corresponding curves. Both EllipSys and NTUA $1 \mathrm{~W}$ predicts similar dynamics on the drag force, figure 4, while the responses from ATEFlap display loops with similar slopes, but slightly wider openings, and thus larger drag variations. The moment coefficient loops have small differences in slopes, as also observed in the steady data; as in the drag case, the ATEFlap code overestimates the dynamic effects and returns wider loops. The curve from NTUA $1 \mathrm{~W}$ is corrupted by some wiggles, which originate from numerical instabilities in the boundary layer solution close to the transition point, and from the transition point traveling along the airfoil surface and switching to a different panel along the suction side.

By further increasing the reduced frequency to $k=0.5$, figure 5 , the unsteady 

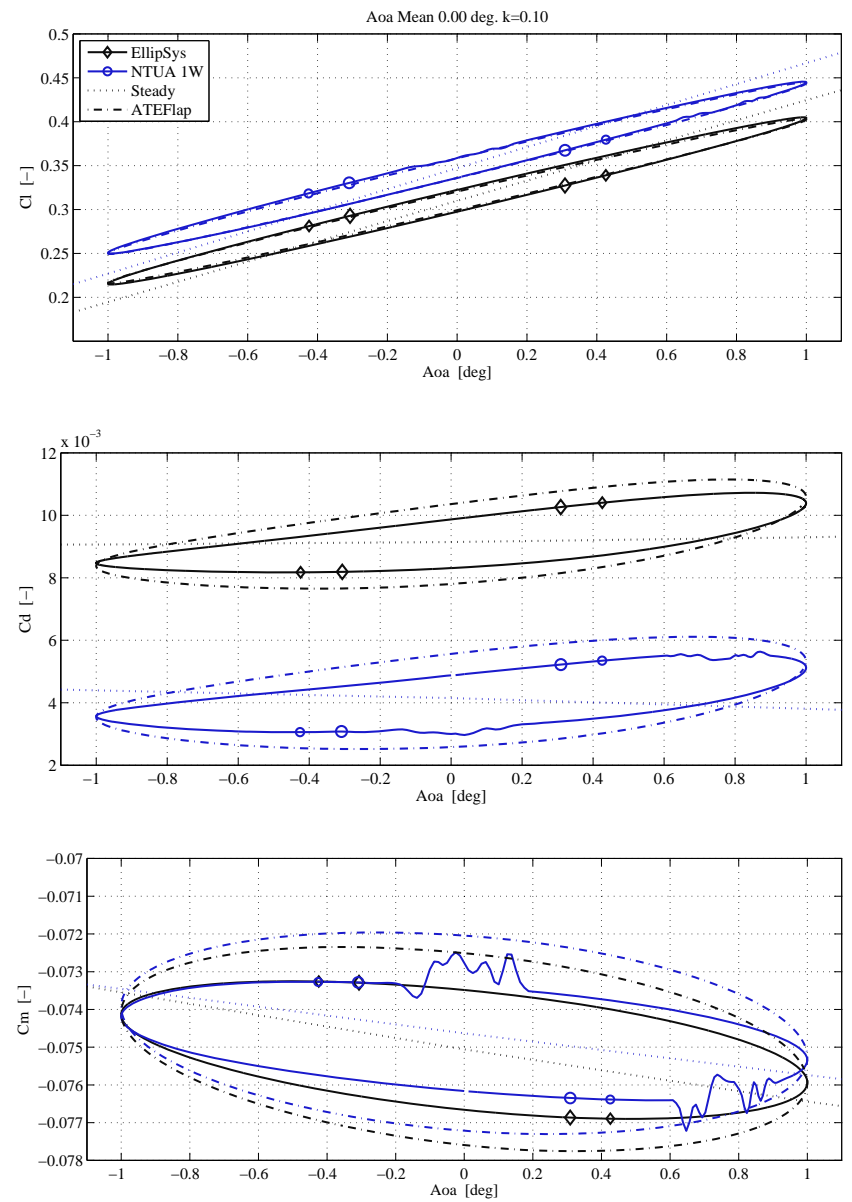

Figure 4: Attached flow conditions. Unsteady aerodynamic response for harmonic pitching motion around $0^{\circ}$ aoa, reduced frequency $k=0.1$. The markers show the direction of the dynamic loops, unfolding from larger to smaller markers. Unless otherwise specified, the ATEFlap loops have the same direction as the corresponding loops from EllipSys or NTUA codes.

lift responses trace loops with lower slopes, wider opening, and direction changed to clockwise; all the methods return similar changes in the dynamics, and the agreement with the ATEFlap is only slightly worse than in the previous case. The unsteady drag response displays a 'knot' in the loops, captured both by EllipSys and NTUA 1W; ATEFlap fails to predict this feature, and returns loops with wider openings in the drag and moment coefficients.

For harmonic oscillations of reduced frequency $k=0.1$ around $12^{\circ}$ aoa, figure 6 , the influence of dynamic stall effects on the aerodynamic responses is evident, as the unsteady lift coefficients reach higher values than their steady counterparts; the effect is well captured by all models. ATEFlap returns loops with similar slope and openings for both steady input sets, and it is in fairly good agreement with EllipSys; NTUA 1W predicts larger lift variations. Apart from the offset observed in the steady data, the dynamics on the drag coefficient predicted by EllipSys and NTUA 1W are in good agreement, whereas ATEFlap 

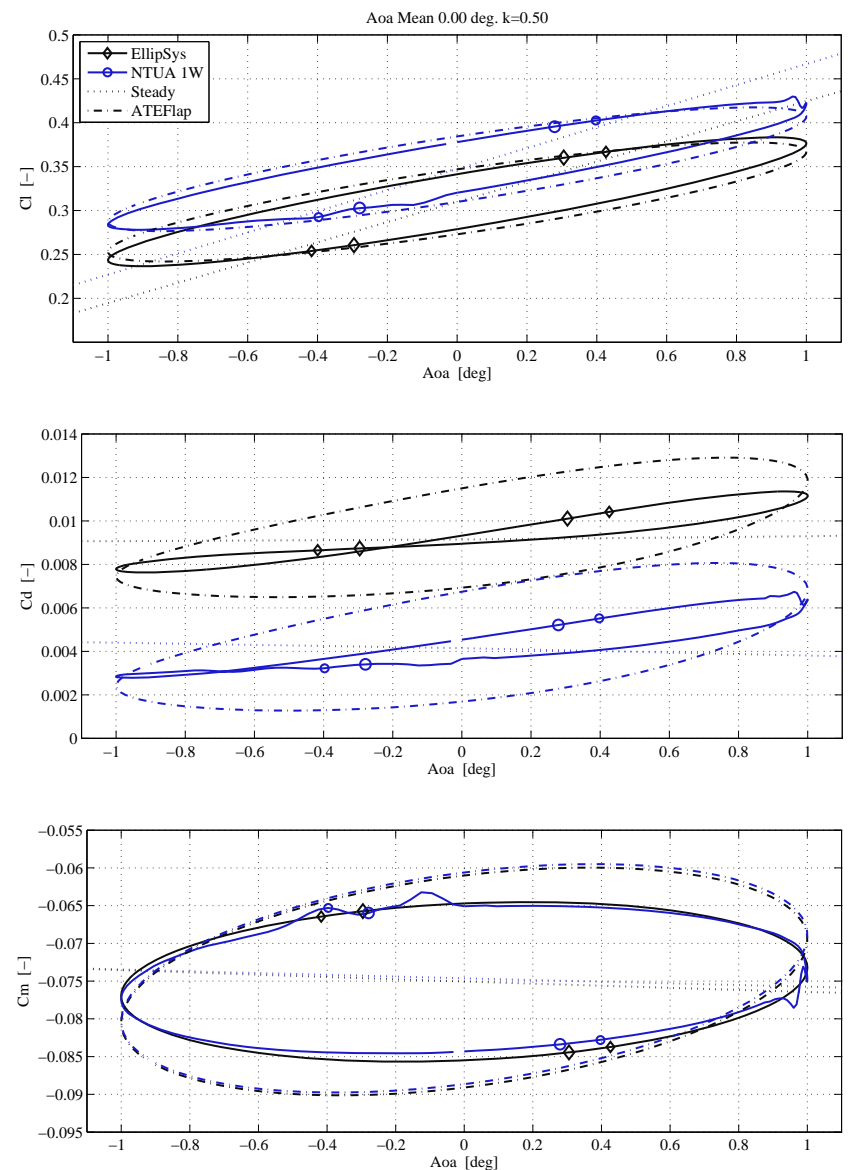

Figure 5: Attached flow, highly unsteady conditions. Unsteady aerodynamic response for harmonic pitching motion around $0^{\circ}$ aoa (attached flow), reduced frequency $k=0.5$. The ATEFlap results trace clockwise loops for the lift and drag, and counterclockwise loops for the moment coefficients.

overestimates the opening and slopes of the loops and, as for lower angles of attack, returns larger drag variations, figure 6 . The wiggles in the drag and moment responses from NTUA $1 \mathrm{~W}$ are again related to numerical instabilities from the transition point travelling and the boundary layer solution in its proximities.

As the angle of attack is increased, flow separation along the airfoil becomes more marked, and the thin-wake assumption is no longer valid; the NTUA $2 \mathrm{~W}$ double-wake implementation is thus necessary to avoid significant biases in the response predicted by the viscous-inviscid interaction method. Figure 7 displays the responses simulated in the challenging case of well developed stalled conditions, harmonic pitch motion occurring around $18^{\circ}$ aoa, with a reduced frequency $k=0.1$, and an amplitude of $\pm 2.5^{\circ}$. Besides the offset already observed in the steady data, the numerical methods EllipSys and NTUA 2W return similar dynamic responses: the unsteady lift loops have steeper slopes than the corresponding steady curves, and displays a total lift variation much higher than the corresponding steady one, due to stall hysteresis effects; on 

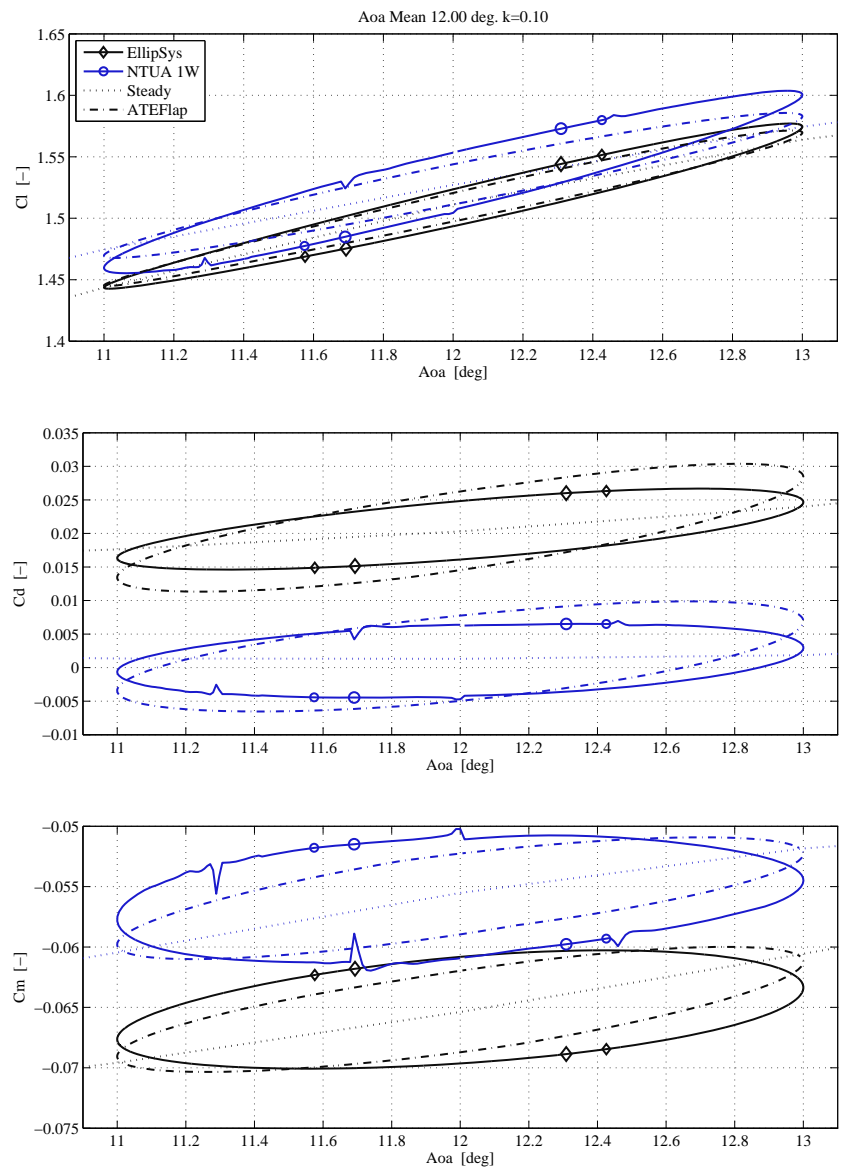

Figure 6: Separation onset. Unsteady aerodynamic response for harmonic pitching motion around $12^{\circ}$ aoa, reduced frequency $k=0.1$. The ATEFlap loops have the same direction of revolution as the corresponding loops from EllipSys or NTUA $1 \mathrm{~W}$.

the contrary, the drag coefficient loops have less steep slopes and smaller drag variations than given by the steady curves, figure 7 .

EllipSys and NTUA 2W display similar high frequencies oscillations in the down stroke response, which are caused by unsteady vortex shedding in the wake of the airfoil, resembling the classic von Karman vortex street behind a cylinder. The agreement with ATEFlap is deteriorated in such highly separated flow conditions. The engineering model predicts correctly the direction of the loops, but has a tendency to under-predict the variations in the lift force, and to over-predict the drag dynamics in comparison to the results of the more complex models. The moment variations predicted by ATEFlap have a similar range as in EllipSys and NTUA 2W, but a different phase, thus yielding to different slopes in the dynamic loops. 

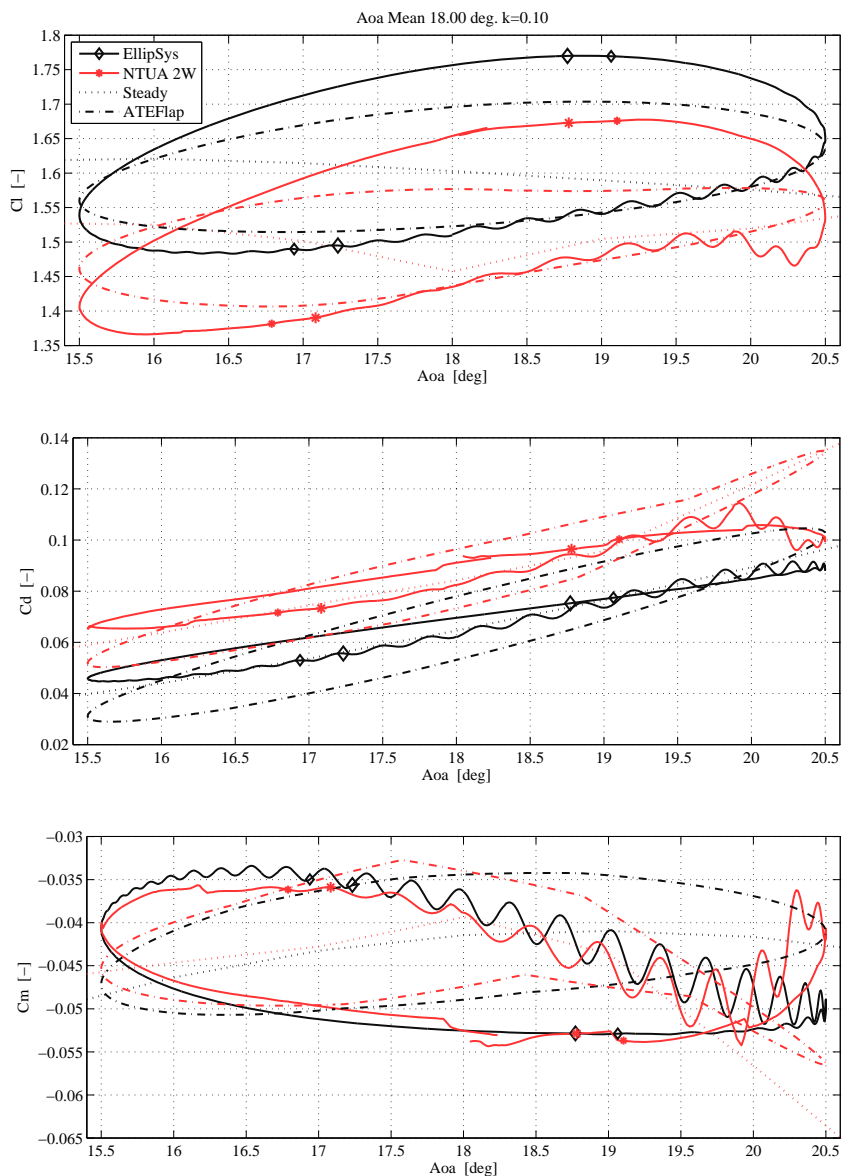

Figure 7: Airfoil in stalled conditions. Unsteady aerodynamic response for harmonic pitching motion around $18^{\circ}$ aoa, reduced frequency $k=0.1$. The ATEFlap loops have the same direction of revolution as the corresponding loops from EllipSys or NTUA $2 W$.

\subsection{Harmonic flap deflections}

The ability of the codes to describe the dynamic effects of the flap motion is determined by comparing the unsteady responses for the airfoil undergoing harmonic flap deflections, while the angle of attack is maintained unchanged; a few representative cases are discussed in the following paragraphs.

The flap deflection generates unsteady aerodynamic forces with dynamics similar to the ones caused by variations in angle of attack. In attached flow, figure 8, the lift force delineates dynamic loops with an effective slope slightly below the steady one; as the deflection frequency increases, the effective slope is further reduced. The ATEFlap engineering model shows excellent agreement with the unsteady lift simulated by the numerical models, figure 8 , while the dynamics on the drag force are slightly over-estimated; a good agreement on the lift force prediction is also maintained at higher reduced frequencies, not shown here. All models agree in predicting moment coefficient responses where the steady variation caused by the flap deflection is by far dominant over the 

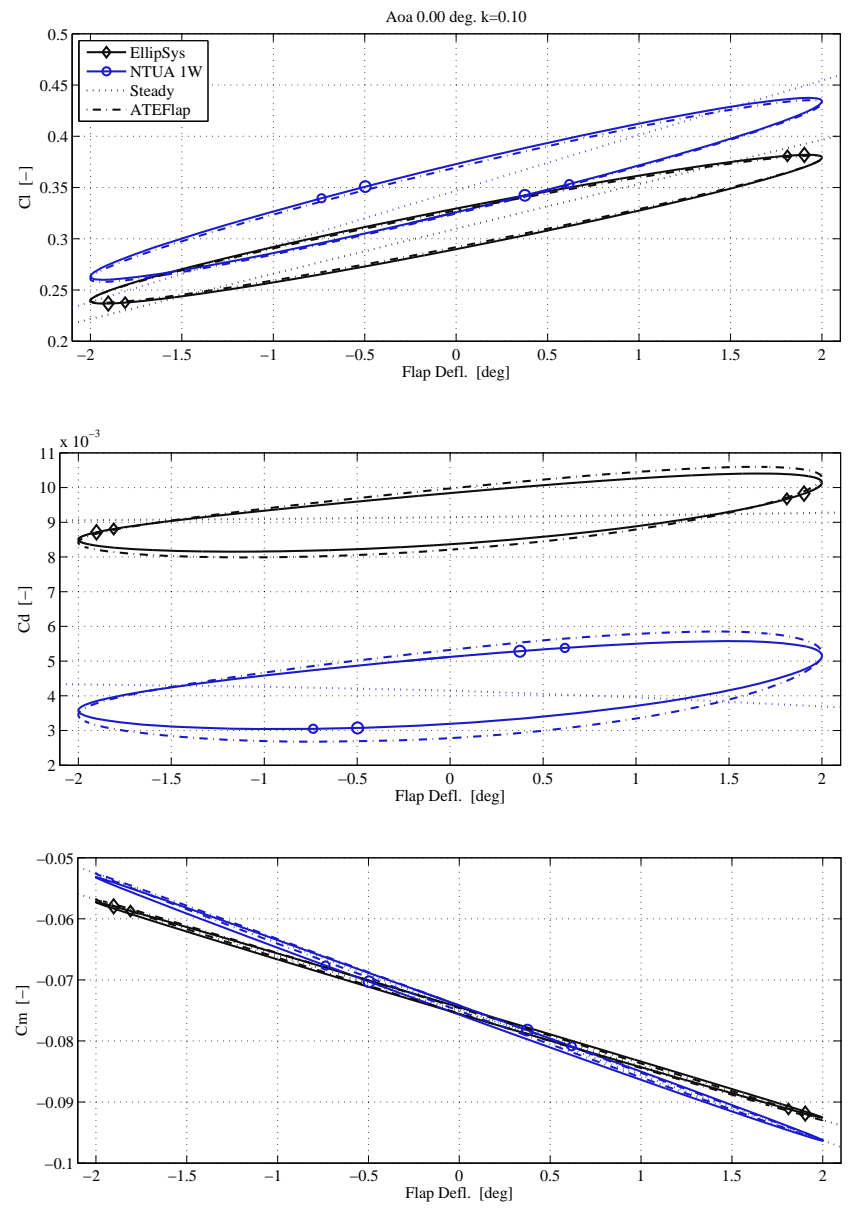

Figure 8: Attached flow conditions. Unsteady aerodynamic response for harmonic flap deflections, angle of attack fixed at $0^{\circ}$ aoa, reduced frequency $k=0.1$. The markers show the direction of the dynamic loops, unfolding from larger to smaller markers. The ATEFlap loops have the same direction of revolution as the corresponding loops from EllipSys or NTUA $1 \mathrm{~W}$

dynamic effects; the loops outlined by the dynamic responses remain very close to their steady curve values, as also predicted by thin-airfoil theory. The offsets between the mean values of the dynamic response loops reflect the difference in the airfoil steady coefficients (see fig. 2), whereas the differences in the slopes are related to the steady coefficient variations given by the flap deflection (see fig. 3).

At higher angles of attack, figure 9, the reduction of the flap effects on the lift force returns both steady curves and dynamic lift loops with less steep slopes than reported in the attached flow case; the reduction is particularly evident on the NTUA $1 \mathrm{~W}$ simulations. All the models, including ATEFlap, display good agreement on the drag response simulations, which maintain similar dynamics to the ones observed at lower angles of attack.

The ATEFlap model, while correctly reproducing the lift dynamics predicted by EllipSys, shows an interesting mismatch in the lift response based on the 

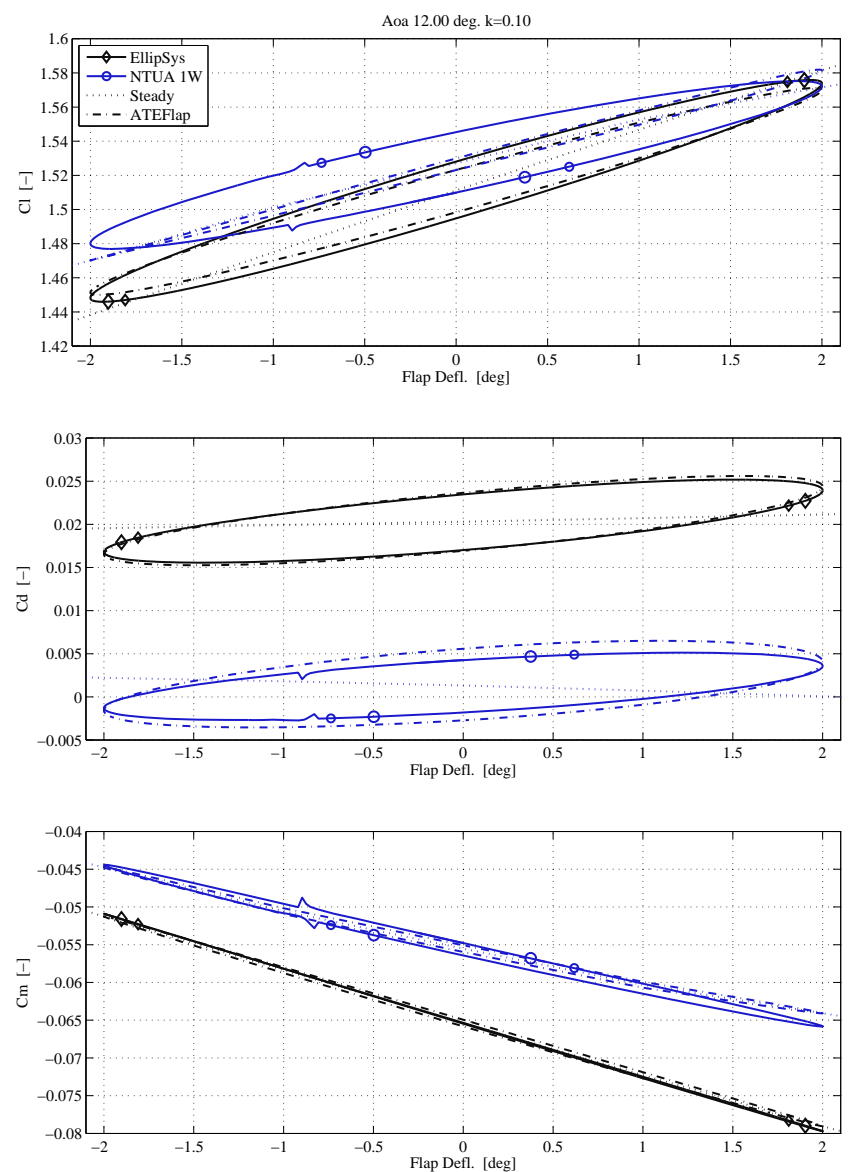

Figure 9: Separation onset. Unsteady aerodynamic response for harmonic flap deflections, angle of attack fixed at $12^{\circ}$ aoa, reduced frequency $k=0.1$. The ATEFlap loops have the same direction of revolution as the corresponding loops from EllipSys; the ATEFlap loop based on NTUA $1 \mathrm{~W}$ data are clockwise both for drag, and lift coefficients.

NTUA 1W steady data: the loop simulated by ATEFlap is much closer to the steady curve and rotates clockwise, figure 9 . The cause of the mismatch roots in the steady input data, where, for higher angles of attack, NTUA 1W predicts a more marked reduction of the steady lift variation achieved by the flap than EllipSys does, see fig. 3(a); the reduction in the flap lift effectiveness is reckoned by the ATEFlap model as caused by flow separation, consequently, the model assumes the degree of flow separation to vary considerably as the flap deflection changes. The lift response predicted by ATEFlap based on the NTUA 1W data at $12^{\circ}$ aoa is thus largely affected by flow separation dynamics, which are not present to such an extent in the corresponding unsteady simulations by NTUA 1W. As the flow separation dynamics are much slower than the attached flow ones, the lift response from ATEFlap and the corresponding NTUA 1W loop display a considerable mismatch. A comparison with experimental data reproducing this particular condition would be of greatest interest.

At an angle of attack of $16^{\circ}$, which is above the maximum lift one, the lift 

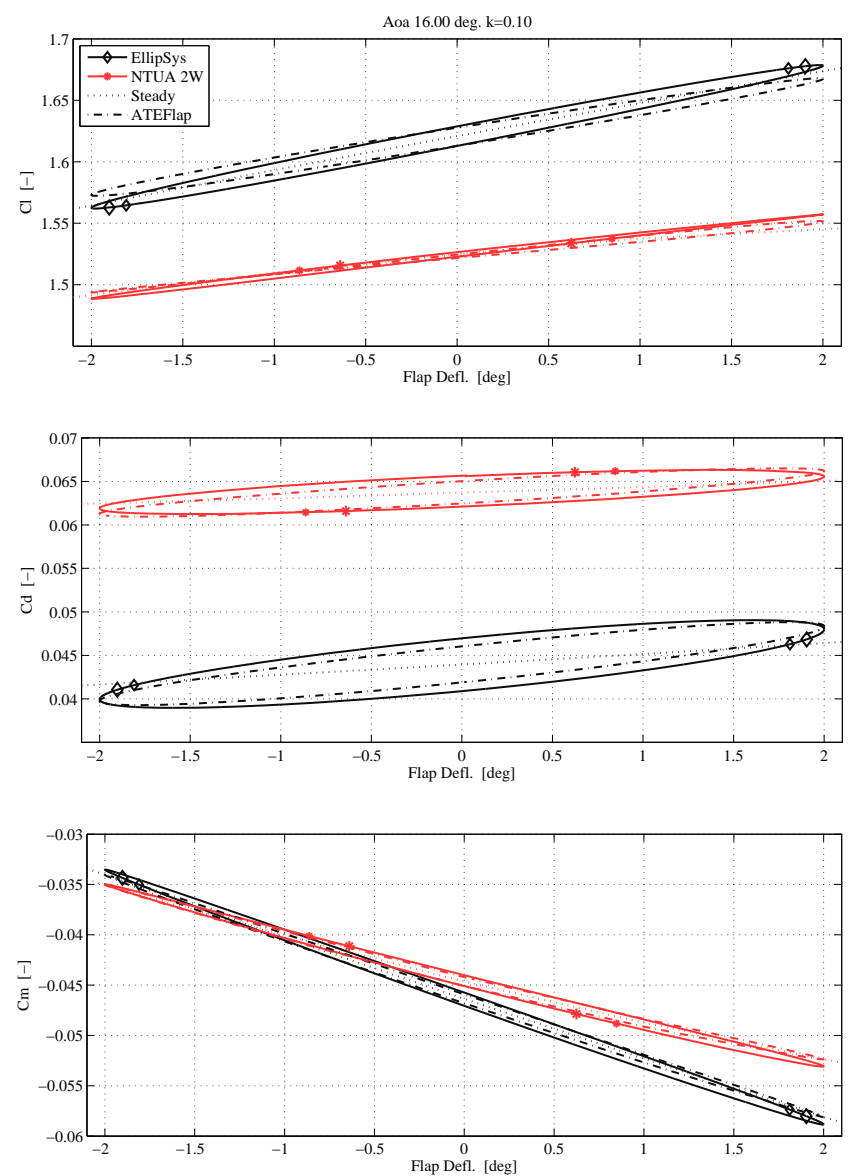

Figure 10: Stalled flow conditions. Harmonic flap deflections, angle of attack fixed at $16^{\circ}$ aoa, reduced frequency $k=0.1$. The ATEFlap loops have the same direction of revolution as the corresponding loops from EllipSys or NTUA $2 \mathrm{~W}$.

response loops get closer to the steady curves, figure 10; the behavior is captured by all models, although ATEFlap returns loops with slightly lower slopes, and thus smaller lift variations. EllipSys and NTUA 2W return unsteady drag loops with similar shapes, and larger openings than at lower angles of attack; the loops are fairly well predicted by the ATEFlap model, which returns similar drag variations, although with less open loops. The moment coefficients still maintain very close to their steady values.

Due to highly stalled conditions, the simulation of the response at $18^{\circ}$ angle of attack and for flap deflections of $\pm 5^{\circ}$ poses the greatest challenges, figure 11 . Both EllipSys and NTUA 2W display high frequency oscillations, again related to the unsteady vortex street shed in the wake of the airfoil. The unsteady response from EllipSys develops around a mean value different from the steady one; offset aside, ATEFlap is able to capture the characteristics of the EllipSys response, and returns loops with similar opening, and the same direction: counter-clockwise for the lift coefficient, and clockwise for the drag. The lift response simulated with NTUA $2 \mathrm{~W}$ outlines instead a clockwise loop, with a 

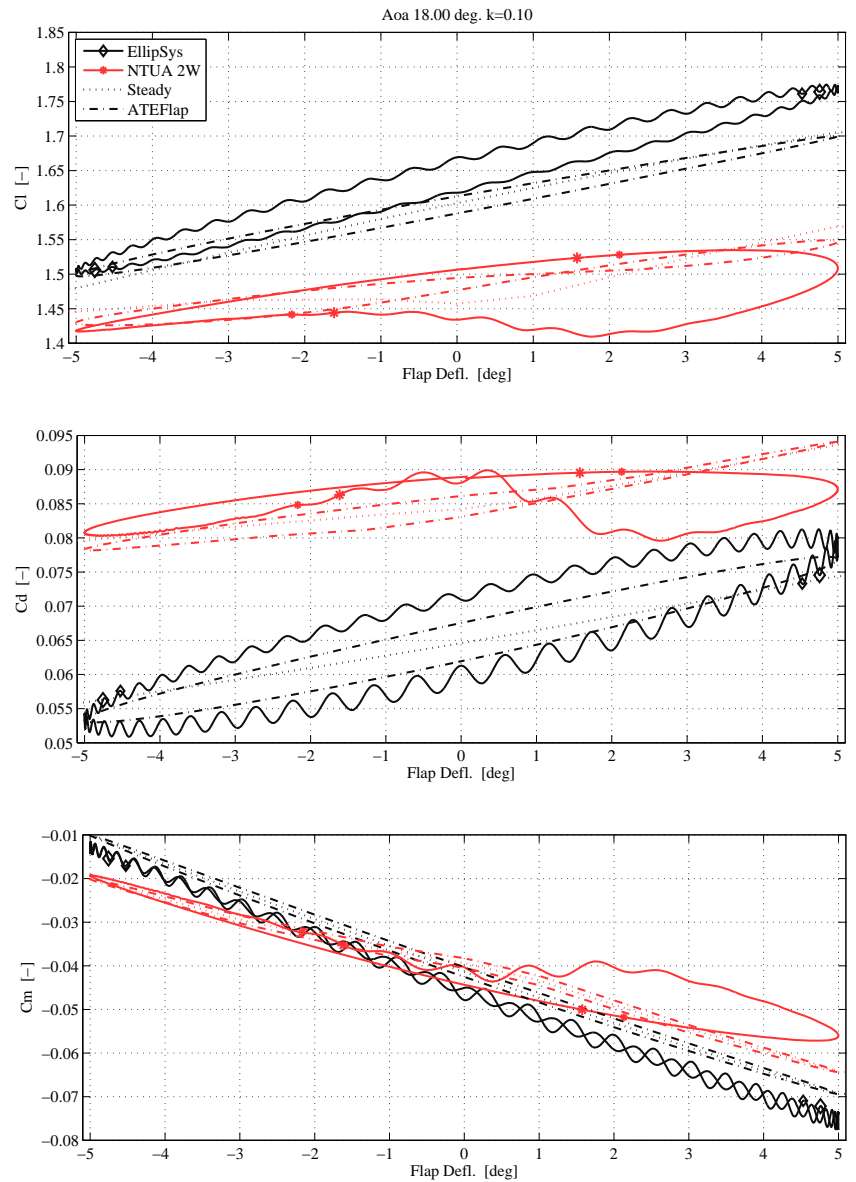

Figure 11: Highly stalled conditions. Harmonic flap deflections, angle of attack fixed at $18^{\circ}$ aoa, flap variations $\pm 5^{\circ}$, reduced frequency $k=0.1$. The ATEFlap loops have the same direction of revolution as the corresponding loops from EllipSys (counter-clockwise $\mathrm{Cl}$, clockwise Cd) or NTUA $2 W$ (clockwise loops).

similar opening for negative flap deflection, but a marked decrease in lift and an increase in drag as the flap starts to move upwards; the behavior is driven by the local deep in the steady $\mathrm{Cl}$ curve around $18^{\circ}$ aoa noted in NTUA $2 \mathrm{~W}$ results, which suggests local stall conditions and gives rise to higher dynamic stall hysteresis effects. ATEFlap returns loops with the same direction, but fails to capture the lift drop, and instead returns a figure-eight loop. The moment coefficients are also affected by high frequency oscillations, but the response is still largely dominated by the steady moment variations.

\section{Conclusion}

The aerodynamic response of an airfoil section with a trailing edge flap has been characterized in steady and unsteady conditions, simulating the effects of changes in angle of attack, and flap deflection. Simulations were carried out 
with three different codes: EllipSys Navier-Stokes solver, NTUA viscous-inviscid interaction method, and the ATEFlap engineering model.

In steady conditions, simulations of the flap effects with EllipSys and the NTUA viscous-inviscid codes outline the same trends: a downward deflection yields an increase in lift, and a downward pitching moment; as the angle of attack is increased, the lift and moment variations brought by the flap deflection decrease, whereas the drag increment increases. Although they describe similar trends, the codes return steady coefficients with different values, a discrepancy which is then reflected throughout the dynamic response simulations.

The unsteady response is characterized by significant differences of the integral aerodynamic forces and moment coefficients from their steady counterparts. In attached flow conditions, harmonic pitching motion returns smaller lift variations, and larger drag and moment variations than returned by a simple look-up of the steady values. The response to harmonic flap deflections is characterized by similar dynamics on the unsteady lift and drag forces, whereas the variations in moment are dominated by the steady component. As flow separation develops along the airfoil, stall dynamics modify the response, and harmonic pitch motions result in unsteady lift variations larger than the steady ones. The effect is not observed in the unsteady lift response from flap deflections, which instead get closer to the steady curves.

A comparison of the characteristics of the unsteady responses simulated by EllipSys and the NTUA 1W code highlights an excellent agreement of the dynamics of integral forces and moments predicted by the two methods in attached flow conditions, both for harmonic pitching motion, and flap deflection. The agreement deteriorates at higher angles of attack, as more complex stalled flow dynamics affect the unsteady response. Nonetheless, the unsteady responses from the two methods still display similar characteristics, and the differences in the dynamic responses produce anyway smaller variations than the ones observed in the steady data.

Similar considerations hold for the ATEFlap engineering model. In attached flow conditions, the model reproduces very well the dynamics of the unsteady lift force, both for pitch and flap deflection variations; at higher angles of attack, the responses simulated by ATEFlap start to diverge from the ones returned by more computationally expensive methods. No effort is made in this study to reduce the differences by re-tuning the parameters $\tau_{P}$ and $\tau_{B}$, which regulate the dynamics of the Beddoes-Leishmann type dynamic-stall model. Concerning the drag force and the aerodynamic moment, ATEFlap seems to slightly over-predict the dynamic effects from changes in the angle of attack, while a closer match is achieved on the unsteady drag and moment responses from flap deflection.

The ATEFlap engineering model has much lower computational requirements than the investigated numerical methods, a quality highly appreciated in aeroelastic simulation tools: the requirements are indicatively 500-800 times lower than EllipSys and 10-15 times lower than the viscous-inviscid interaction methods. Yet, in spite few differences from the response returned by the more complex methods, the ATEFlap model proved able to describe with sufficient accuracy the relevant dynamics of the unsteady forces and moments, originated both from airfoil motion and flap deflection. Considering also that the outer part of the blades on a pitch regulated turbine operates most of the time in attached flow conditions, the ATEFlap model is judged suitable for implementation in a BEM-based aeroelastic simulation tool. The aerodynamic response for an airfoil 
in highly stalled conditions computed by the ATEFlap model -as probably by any other engineering dynamic stall model- should be instead considered with greater circumspection.

The ATEFlap model, as many of the models used in aeroelastic simulations, relies on steady input data, which are generated by other methods. The simulations have shown that discrepancies in the steady input data might affect the aerodynamic response to a much higher degree than differences in the unsteady force modeling. In this sense, future work should consider a comparison between the steady aerodynamic responses predicted by the numerical methods and experimental data, including the effects of trailing edge flap deflections. Experimental data for the unsteady aerodynamic responses originated by flap deflection would certainly also be of great interest.

\section{Acknowledgment}

This work was partly funded by the European Commission under contract SES6 019945 (UpWind Integrated Project).

\section{References}

[1] Barlas TK, van Kuik G. State of the art and prospectives of smart rotor control for wind turbines. Journal of Physics: Conference Series 2007; 75(1):012080 (20 pp.).

[2] Johnson SJ, Baker JP, van Dam CP, Berg D. An overview of active load control techniques for wind turbines with an emphasis on microtabs. Wind Energy Mar 2010; 13(2-3):239-253, doi:10.1002/we.356.

[3] Buhl T, Gaunaa M, Bak C. Potential load reduction using airfoils with variable trailing edge geometry. Transactions of the ASME. Journal of Solar Energy Engineering 2005; 127(4):503-516.

[4] Barlas TK, van der Veen GJ, van Kuik GA. Model predictive control for wind turbines with distributed active flaps: incorporating inflow signals and actuator constraints. Wind Energy 2011; doi:10.1002/we.503.

[5] Castaignet D, Poulsen NK, Buhl T, Wedel-Heinen JJ. Model predictive control of trailing edge flaps on a wind turbine blade. Proceedings of the American Control Conference 2011, San Francisco, CA, USA, 2011.

[6] Wilson DG, Berg DE, Barone MF, Berg JC, Resor BR, Lobitz DW. Active aerodynamic blade control design for load reduction on large wind turbines. European Wind Energy Conference, Marseille, France, 2009; 26(19).

[7] Troldborg N. Computational study of the Ris $\varnothing-B 1-18$ airfoil with a hinged flap providing variable trailing edge geometry. Wind Engineering 2005; 29(2):89-113.

[8] van Dam CP, Chow R, Zayas JR, Berg DE. Computational investigations of small deploying tabs and flaps for aerodynamic load control. Journal of Physics: Conference Series 2007; 75(1):012 027-1-10. 
[9] Heinz J, Sørensen NN, Zahle F. Investigation of the load reduction potential of two trailing edge flap controls using CFD. Wind Energy 2011; 14(3):449 462, doi:10.1002/we.435.

[10] Ng BF, Palacios R, Graham JMR, Kerrigan EC. Robust control synthesis for gust load alleviation from large aeroelastic models with relaxation of spatial discretization. Conference proceedings, European Wind Energy Conference 2012, Copenhagen, Denmark, 2012.

[11] Ramos Garcia N. Quasi-3d aerodynamic code for analyzing dynamic flap response. Ph.D. thesis, Technical University of Denmark, Department of Mechanical Engineering, Lyngby, Denmark Apr 2011.

[12] Riziotis VA, Voutsinas SG. Aero-elastic modelling of the active flap concept for load control. Conference proceedings, European Wind Energy Conference 2008, Marseille, France, 2008.

[13] Lackner MA, van Kuik G. A comparison of smart rotor control approaches using trailing edge flaps and individual pitch control. Wind Energy Mar 2010; 13(2-3):117-134, doi:10.1002/we.353.

[14] Barlas TK, Van Kuik GAM. Aeroelastic modelling and comparison of advanced active flap control concepts for load reduction on the upwind 5MW wind turbine. European Wind Energy Conference, Marseille, France, 2009; $16(19)$.

[15] Andersen PB, Gaunaa M, Bak C, Hansen MH. A dynamic stall model for airfoils with deformable trailing edges. Wind Energy 2009; 12(8):734-751, doi:10.1002/we.326.

[16] Riziotis VA, Voutsinas SG. Dynamic stall modelling on airfoils based on strong viscous-inviscid interaction coupling. International Journal for $\mathrm{Nu}$ merical Methods in Fluids 2008; 56(2):185-208, doi:10.1002/fld.1525.

[17] Bergami L, Gaunaa M. ATEFlap aerodynamic model, a dynamic stall model including the effects of trailing edge flap deflection. Technical Report $R$-1792(EN), Risoe National Laboratory. Technical University of Denmark, Roskilde, Denmark Feb 2012.

[18] Gaunaa M, Riziotis VA, Timmer N, Snel H, Andersen PB, Heinz J. Comparison of code performance for computation of unsteady $2 \mathrm{D}$ aerodynamics of airfoils with deformable trailing edges. Technical Report D2.5, UpWind - Integrated Wind Turbine Design Feb 2011.

[19] Michelsen JA. Basis3D - a platform for development of multiblock PDE solvers. Technical Report AFM 92-05, Technical University of Denmark 1992.

[20] Michelsen JA. Block structured Multigrid solution of 2D and 3D elliptic PDEs. Technical Report AFM 94-06, Technical University of Denmark 1994.

[21] Sørensen N. General purpose flow solver applied to flow over hills. Technical Report Risoe-R-827(EN), Risoe National Laboratory, Roskilde (DK) 1995. 
[22] Menter FR. Two-equation eddy-viscosity turbulence models for engineering applications. AIAA journal 1994; 32(8):1598-1605.

[23] Voutsinas SG, Riziotis VA. A viscous-inviscid interaction model for dynamic stall simulations on airfoils. Proceedings of 37th Aerospace Sciences Meeting and Exhibit, vol. 99-0038, AIAA: Reno, Nevada, 1999.

[24] Riziotis VA, Voutsinas SG, Politis E, Chaviaropoulos P. Stability analysis of parked wind turbine blades using a vortex model. Proceedings of the Science of Making Torque from the Wind, Heraklion, Greece, 2010.

[25] Gaunaa M. Unsteady two-dimensional potential-flow model for thin variable geometry airfoils. Wind Energy 2010; 13(2-3):167-192, doi:10.1002/ we.377.

[26] Bergami L, Gaunaa M, Heinz J. Indicial lift response function: an empirical relation for finite-thickness airfoils, and effects on aeroelastic simulations. Wind Energy 2013; 16(5):681-693, doi:10.1002/we.1516.

[27] Hansen MH, Gaunaa M, Madsen HA. A Beddoes-Leishman type dynamic stall model in state-space and indicial formulations. Technical Report $R$ 1354(EN), Risoe National Laboratory, Roskilde (DK) 2004. 